University of New Hampshire

University of New Hampshire Scholars' Repository

Physics Scholarship

Physics

$12-1988$

\title{
On the possibility of quasi-static convection in the quiet magnetotail
}

Margaret G. Kivelson

University of California - Los Angeles

Harlan E. Spence

University of New Hampshire, harlan.spence@unh.edu

Follow this and additional works at: https://scholars.unh.edu/physics_facpub

Part of the Physics Commons

\section{Recommended Citation}

Kivelson, M. G. and H. E. Spence, On the possibility of quasi-static convection in the quiet magnetotail, Geophys. Res. Lett., 15, 1541, 1988.

This Article is brought to you for free and open access by the Physics at University of New Hampshire Scholars' Repository. It has been accepted for inclusion in Physics Scholarship by an authorized administrator of University of New Hampshire Scholars' Repository. For more information, please contact Scholarly.Communication@unh.edu. 
GEOPHYSICAL RESEARCH LETTERS, VOL. 15, NO. 13, PAGES 1541-1544, DECEMBEER 1988

\title{
ON THE POSSIBILITY OF QUASI-STATIC CONVECTION IN THE QUIET MAGNETOTAIL
}

\author{
Margaret G. Kivelson and Harlan E. Spence
}

Institute of Geophysics and Planetary Physics and Department of Earth and Space Sciences, UCLA

\begin{abstract}
The magnetotail is known to serve as a reservoir of energy transferred into the terrestrial magnetosphere from the solar wind. In principle, the stored energy can be dissipated impulsively, as in a substorm, or steadily through the process of steady adiabatic plasma convection. However, some theoretical arguments have suggested that quasi-static adiabatic convection cannot occur throughout the magnetotail because of the structure of the magnetic field. Here we reexamine the question. We show that in a magnetotail of finite width, downtail pressure gradients depend strongly on the ratio of the potential across half the tail to the ion temperature in the far tail $\left(60 R_{E}\right)$. For pertinent quiet time ratios $(-3)$, a Tsyganenko quiet-time magnetic field model is consistent with steady convection.
\end{abstract}

\section{Introduction}

Phenomena in the terrestrial magnetosphere can be described by the equations of magnetohydrodynamics if the focus is on length scales large compared with particle gyroradii and time scales long with respect to the periods of particle orbits. For slow, steady state convection, static tail-like configurations would appear to satisfy the conditions required for the MHD approximation other than in a local region near the distant neutral line and in the immediate vicinity of various boundaries. It was, therefore, somewhat unexpected that Erickson and Wolf [1980] found reasons to question whether the earth's magnetotail ever attains a steady state in the presence of slow convection. From analysis of standard models of the magnetic field they concluded that "steady, adiabatic convection probably cannot occur throughout a closed-magnetic-field-line region that extends into a long magnetotail." The significance of this proposal is profound, because if no quasi-static solution exists, substorms or analogous temporal variations would be driven by even rather slow convection in the magnetotail.

We have reexamined the arguments that lead Erickson and Wolf [1980; see also Erickson, 1984, 1985; Schindler and Birn, 1982; Hau et al., 1988] to question the possibility of a tail stable to slow plasma convection. The analysis relies on a model of the magnetic field to provide the gradient of flux tube volume, a quantity that is ill-constrained by observations. For studies of the quiet tail, we selected a magnetic field model (not available to Erickson and Wolf) valid for low levels of activity [Tsyganenko, 1987]. We show that both the improved field model and corrections for the finite width of the tail modify the results previously obtained. Including corrections for finite tail width which were previously underestimated [Erickson, 1985, hereinafter referred to as E-85], we find a plasma sheet pressure that is not inconsistent with the lobe magnetic pressure. We do not find extremely large plasma pressure near $10 \mathrm{R}_{\mathrm{E}}$ [Erickson, 1984; Hau et al., 1988] and do not expect an associated "deep minimum" to form in the magnetic field strength. We see no reason to anticipate that the tail would become unstable in the presence of the level of slow

Copyright 1988 by the American Geophysical Union.

Paper number 88GL03940.

0094-8276/88/88GL-03940\$03.00 convection expected during intervals of low geomagnetic activity.

\section{Background}

Erickson and Wolf [1980, referred to as EW-80] used the variation of flux tube volume for several models of magnetotail fields to calculate the pressure of adiabatically convected plasma in the magnetotail. If pitch angle scattering maintains isotropy, the pressure, $\mathrm{p}$, along a drift trajectory satisfies

$$
\mathrm{p} \propto \mathrm{V}_{\mathrm{e}}^{-5 / 3}
$$

where $V_{e}$ is the volume of a flux tube passing through the center of the plasma sheet at a distance $x_{e}$ down the tail.

For slow convection, the plasma sheet pressure gradient must balance the $\mathbf{j} \times \mathbf{B}$ stress of the magnetic field. Beyond about $15 \mathrm{R}_{\mathrm{E}}$ the essentially planar geometry of the tail is consistent with neglect of the small contribution of lobe magnetic field curvature. The validity of this approximation has been demonstrated by Spence et al. [1988a] who find that the gradient of observed plasma sheet pressure equals the gradient of lobe magnetic pressure beyond $-15 R_{\mathrm{E}}$. Then force balance in the $\mathrm{z}$ direction (normal to the plasma sheet) requires that the plasma sheet pressure, $\mathrm{p}\left(\mathrm{x}_{\mathrm{e}}\right)$, balance the lobe magnetic pressure, $P_{L}\left(x_{e}\right)=B_{L}\left(x_{e}\right)^{2 / 2} \mu_{0}$ where $B_{L}$ is the lobe magnetic field. In the tail, $B_{L} \propto x^{-L}$. Thus the ratio $p$,

$$
\mathrm{p}=\left[\mathrm{p}\left(\mathrm{x}_{\mathrm{e}}\right) / \mathrm{P}_{\mathrm{L}}\left(\mathrm{x}_{\mathrm{e}}\right)\right] /\left[\mathrm{p}\left(\mathrm{x}_{\mathrm{o}}\right) / \mathrm{P}_{\mathrm{L}}\left(\mathrm{x}_{\mathrm{o}}\right)\right]=\left(\mathrm{V}_{\mathrm{o}} / \mathrm{V}_{\mathrm{e}}\right)^{5 / 3}\left(\mathrm{x}_{\mathrm{e}} / \mathrm{x}_{\mathrm{o}}\right)^{2 \mathrm{~L}}
$$

must equal 1 for all $\mathrm{x}_{\mathrm{e}}$ independent of distance beyond about $15 R_{E}$. EW-80, citing Behannon [1968] and Mihalov and Sonett [1968], select $L=-0.6 \pm 0.2$, a point to which we will return. With flux tube volumes obtained from various field models [Beard, 1979; Olson and Pfitzer, 1974; Voigt, 1981] and with $x_{0}=60 R_{E}$ ( $\rho$ is 1 by definition at $x_{0}$ ), they find that $\rho$ takes the values 3 to $3.5,4$ to 9.5 , and 13 to $>200$ for $x_{e}=30$, 20 , and $10 R_{E}$, respectively. Adding curvature forces, they find that force balance remains inconsistent with pressures calculated from equation (1).

Since 1980 the concept of a steady state configuration of the magnetotail has been further questioned [Schindler and Birn, 1982; Tsyganenko, 1982; Erickson, 1984, 1985; Hau et al., 1988 ]. If the convected plasma pressure becomes sufficiently large near $10 \mathrm{R}_{\mathrm{E}}$, a minimum develops in the magnetic field intensity and this could make the system unstable to reconnection. Under such circumstances, a steady state of the slowly convecting plasma seems unlikely.

The arguments summarized above rest on several simplifying assumptions. The most significant are that losses associated with inward convection are negligible and that the tail system can be treated as two dimensional. Losses through strong pitch angle diffusion could reduce the rate of pressure increase as the flux tubes convect towards the earth, but E-85 found the loss rate insufficient to modify the near-earth pressure significantly. This result is supported by recent studies [Spence et al., 1988b] of the Tsyganenko and Usmanov [1982] and Tsyganenko [1987] field models (referred to as TU and T87, respectively). E-85 also concluded that corrections for the finite width of the tail would not reduce the pressure significantly in the vicinity of the midnight meridian. Tsyganenko [1982] suggested that particle losses and 
associated self-consistent changes in field structure could account for shallower pressure gradients, but he provided only qualitative arguments.

\section{Magnetotail Stresses in Phenomenological MHD Field Models}

Analyses of stress balance in the magnetotail have for the most part been based on two dimensional models required for computational tractability. Insight into the behavior of a more realistic three dimensional magnetotail has been obtained by requiring stress balance in the $\mathrm{TU}$ and $\mathrm{T} 87$ field models that represent actual measured magnetospheric fields. These models are parametrized by levels of geomagnetic activity.

Spence et al. [1987] found that the TU quiet time near-tail model is consistent with static MHD between 6.5 and $12 R_{E}$ for physically reasonable pressure profiles. Assuming that the bulk of the plasma pressure is contributed by ions, Spence et al. [1988a] obtained statistical averages of observed plasma sheet pressure near $0000 \mathrm{LT}$ between 12 and $30 \mathrm{R}_{\mathrm{E}}$ from ISEE-2 data. They found that the pressure, both measured and inferred from the TU and T87 models, satisfies approximately

$$
\mathrm{pV}^{2 / 3}=\text { const. }
$$

between 6.5 and $30 R_{E}$. Schindler and Birn (1982) found this same dependence. Although the near tail pressure does not satisfy equation (1), the existence of a self-consistent static solution suggests that the pressure variation may be imposed through slow convection. For this reason we decided to reassess previous evidence for inconsistency.

\section{Reassessment of Stability Arguments}

In this section we use the T87 field model to model flux tube volumes. We show that beyond $-30 R_{E}$ for a reasonable choice of the lobe field gradient, $\rho$ remains less than 1.5, i.e., consistent with $p=1$ to within uncertainties of models and field fits. Inward of $30 R_{E}$, the ratio $\rho$ continues to grow and becomes unacceptably large in a two dimensional magnetotail of infinite width but $\rho$ does not increase excessively if corrections for the finite width of the tail are included.

Pressure balance beyond $30 \mathrm{RE}$. Let us examine the right hand side of equation (2) and see whether it yields $p \cong 1$ beyond -30 $R_{E}$. The critical parameters are the variation of flux tube volume with distance, and the exponent, $L$, representing the rate of decrease of the equatorial field strength.

We adopt the quiet $\left(\mathrm{K}_{\mathrm{P}}=0,0+\right) \mathrm{T} 87$ model ("long" version) near the midnight meridian for $0^{\circ}$ dipole tilt and assume no yvariation. Figure 1 shows flux tube volumes vs. $x$ along the midnight meridian for the models examined by EW-80 and for the T87 model that we adopt. Discrepancies greater than $20 \%$ are apparent; in addition, volumes from T87 models pertinent to different $\mathrm{KP}$ vary by more than $20 \%$. We, therefore, argue that the uncertainty of the flux tube volumes is of order 20 $40 \%$.

Equation (1) and the T87 model specify the $x$-dependence of adiabatic plasma pressure, plotted in Figure 2 and labeled po. The field pressure at $60 R_{E}$ was used for normalization. Between 30 and $60 \mathrm{R}_{\mathrm{E}}, \mathrm{dV} / \mathrm{dx}$ is larger for the other models included in Figure 1, so the pressure change in the other models (not plotted) is greater. Figure 2 also shows the lobe magnetic pressure, $\mathrm{P}_{\mathrm{L}}$. We analyzed published lobe field data [Mihalov et al., 1968; Mihalov and Sonett, 1968; and Behannon, 1968] to determine L. We find that Behannon's [1986] data can be fitted to $L=-0.68 \pm 0.07$ and Mihalov et al. [1968] give $\mathrm{L}=-0.798 \pm 0.028$ with larger values of $\mathrm{L}$ more representative of quiet conditions. Details of this argument will be published elsewhere [Spence et al., 1988b]. EW-80 used $\mathrm{L}=0.6$ but we take the faster falloff because it is valid for

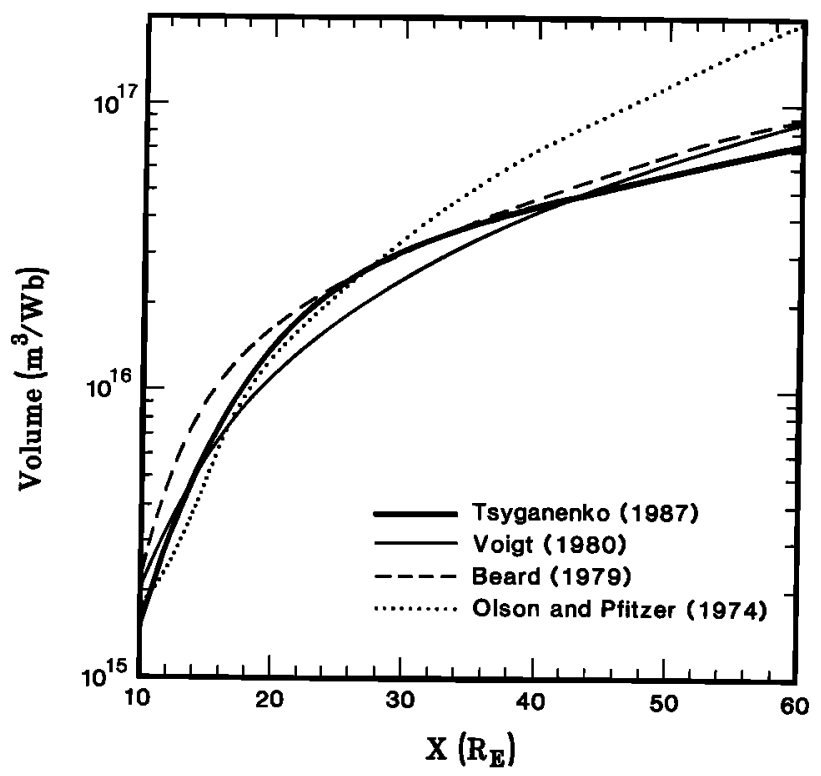

Fig. 1. Flux tube volume vs. down-tail distance, $x$, in the midnight meridian for several model magnetic fields.

quiet times and is well within the uncertainty of the data. Tailward of $30 R_{E}$, we find that $p<1.5$ for the T87 model, consistent with $p=1$ to within the joint uncertainty of the lobe field fit and the field model. (Note that the apparent discrepancy is emphasized because we normalized at $60 \mathrm{R}_{\mathrm{E}}$.) Thus we think that the stability problem identified by EW-80 in the region beyond $30 R_{E}$ at times of low activity is probably not significant.

Modifications related to finite tail width. Let us next consider the essential ways in which the actual three dimensional structure of the tail modifies two dimensional results. Two dimensional treatments assume that the source plasma is present at some large distance, say $\sim 60 R_{F}$, down the tail and that both the plasma parameters and the field depend on down-

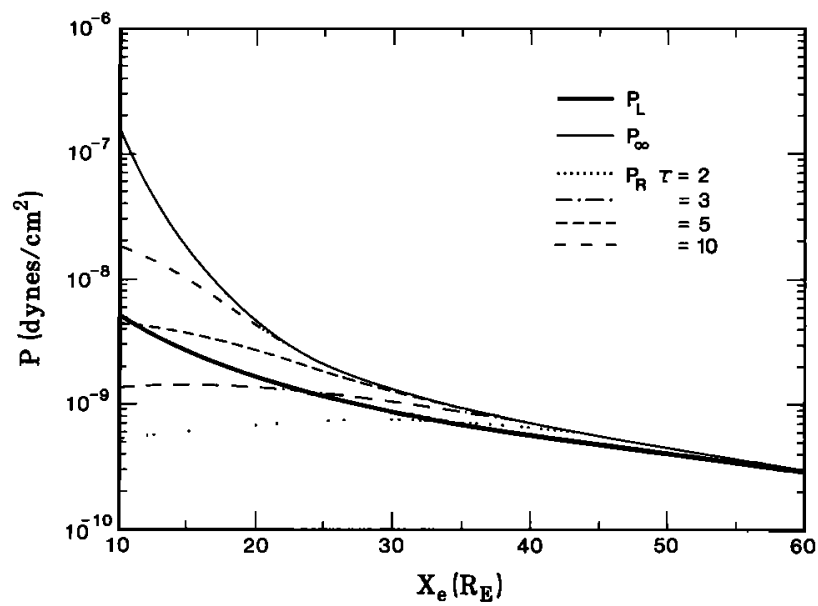

Figure 2. Pressure versus distance from earth in the center of the plasma sheet. $P_{L}$ is a fit to observed lobe magnetic pressure (see text). $p_{\infty}$ is plasma pressure from equation (1). $\mathrm{p}_{\mathrm{R}}$ curves, parametrized by $\tau=\mathrm{qER} / \mathbf{k T}$, are plasma pressures corrected for finite tail width from equations (9) and (10). Flux tube volumes were obtained from the T87 model. Curves have been normalized to the lobe magnetic pressure at $60 R_{E}$. 
tail distance $\left(\mathrm{x}_{\mathrm{e}}\right)$ and distance along the normal to the plasma sheet $(z)$ but not on the cross-tail coordinate, $y$. This means that in the near tail the axially symmetric internal field of the earth is not properly represented and the azimuthal drift of plasma is not accurately modeled, but these may not be critical shortcomings.

Beyond about $40 R_{E}$, the assumption that pressure may be calculated from source plasma distributions uniform across the tail appears to us to be appropriate. However, in calculating the pressure closer to earth, the finite cross-tail width of the region that contains the source plasma should not be ignored. This is because finite energy plasma necessarily is displaced in $y$ as a result of gradient and curvature drifts; the $y$ displacement increases monotonically with inward convection. Assuming that the dawnside low-latitude boundary layer is a negligible source of plasma sheet ions, particles can be assumed to be present at $x_{e}$ on the midnight meridian only if their drift orbits link them with source regions in the distant tail within the actual tail boundaries in y. Fully two-dimensional treatments that ignore this point overestimate the pressure close to the earth by tacitly assuming that sources in the distant tail are unbounded in $y$. A quantitative treatment is required to assess the importance of the effect.

We assume the two dimensional magnetic field geometry illustrated in Figure 2 of T87 and assume no y variation. The source plasma is a maxwellian characterized by density $n_{0}$ and temperature $T_{O}$ at a downtail distance of $60 R_{E}$. The model is quite insensitive to the exact distance selected but extremely sensitive to the assumed source temperature.

We follow the E-85 analysis of the drift of a part of the phase space distribution with initial energy $W_{0}$ on a flux tube of volume $V_{0}$ at $x_{0}$. Sunward convection is produced by a uniform electric field $\mathbf{E}$ in the y-direction and the tail extends from $y=-R$ to $+R$. If pitch angle scattering is invoked to maintain isotropy, the particles reach $x_{e}$ with energy

$$
\mathrm{W}_{\mathrm{e}}=\mathrm{W}_{\mathrm{o}}\left[\mathrm{V}\left(\mathrm{x}_{\mathrm{e}}\right)^{1-\gamma / \mathrm{V}_{0}}{ }^{1-\gamma}\right]
$$

where $\gamma$ is the polytropic index. Energy conservation requires

$$
-q E y+W_{0}\left[V\left(x_{e}\right)^{1-\gamma / V_{0}}{ }^{1-\gamma}\right]=-q E y_{0}+W_{0}
$$

where $q$ is the particle charge and the right side is evaluated at $\left(x_{0}, y_{0}\right)$ within the source plasma. We assume that pressure is carried principally by ions and take $q>0$. On the midnight meridian $(y=0)$, equation $(5)$ reduces to

$$
\mathrm{W}_{\mathrm{o}}=-\mathrm{qE} \mathrm{y}_{\mathrm{o}} /\left[\left[\mathrm{V}_{\mathrm{o}} / \mathrm{V}\left(\mathrm{x}_{\mathrm{e}}\right)\right]^{2 / 3}-1\right\}
$$

where $\gamma$ has been set to $5 / 3$ as in equation (1). An equivalent equation can be found in E-85, though a typographical error has been corrected. For a fixed $x_{e}<x_{0}, W_{0}$ is bounded by $\mathrm{W}_{0} \max$, its value for $-\mathrm{y}_{0}=\mathrm{R}$ at the tail boundary. Evidently,

$$
\mathrm{W}_{0} \max =\mathrm{qE} R /\left[\left[\mathrm{V}_{\mathrm{\alpha}} / \mathrm{V}\left(\mathrm{x}_{\mathrm{e}}\right)\right]^{2 / 3}-1\right\}
$$

and for finite $\mathrm{E}, \mathrm{W}_{0}$ max goes to $\infty$ with the tail width.

To obtain the pressure at $\mathrm{x}_{e}$, we sum contributions from particles of all allowed energies to evaluate the second moment of the distribution function. The distribution function $f_{e}$ at $x_{e}$ is obtained by Liouville's theorem from the distribution function $f_{0}$ (normalized to 1 ) at the plasma source. Only particles with $\mathrm{W}_{0}<\mathrm{W}_{0} \max$ reach $\mathrm{x}_{\mathrm{e}}$ so an upper limit to the velocity space integration, set by $v_{M}=\left(2 W_{0} \max / m\right) 1 / 2$, cuts off the high energy tail of the distribution. Relative to the $R=\infty$ case, the flux tube content decreases only slightly but the plasma effectively "cools" and this reduces the pressure. Following E85 , we find

$$
\frac{p_{e, R}}{p_{\infty}}=\left(\frac{v_{0}}{V}\right) \frac{\int_{0}^{v_{M}} f_{e}(v) v^{4} d v}{\int_{0}^{\infty} f_{0}(v) v^{4} d v}
$$

where $\mathrm{p}_{\mathrm{e}, \mathrm{R}}\left(\mathrm{p}_{\infty}\right)$ is the pressure at $\mathrm{x}_{\mathrm{e}}$ (at midnight) for a tail of width $2 R(\infty)$.

For a maxwellian distribution, $f_{o}(v)$, with $v_{T h}^{2}=2 k T d / m$, equation (8) yields

$$
\mathrm{p}_{\mathrm{e}, \mathrm{R}} / \mathrm{p}_{\infty}=\operatorname{erf}(\zeta)-2 \zeta\left(3+2 \zeta^{2}\right) \exp \left(-\zeta^{2}\right) / 3(\pi)^{1 / 2}
$$

where $\operatorname{erf}(\zeta)$ is the error function and

$$
\zeta^{2}=\left(v_{M} / \mathrm{Th}_{h}\right)^{2}=\mathrm{qE} \mathrm{R} /\left[\left[\mathrm{V}_{\mathrm{O}} / \mathrm{V}\left(\mathrm{x}_{\mathrm{e}}\right)\right]^{2 / 3}-1\right] \mathbf{k} \mathrm{T}_{\mathrm{o}}
$$

As $p_{\infty}$ corresponds to the pressure in a fully two-dimensional treatment, $p_{e, R} / p_{\infty}$ quantifies the effects of a finite tail.

The variation of pressure with $\mathrm{V}$ is model-independent even though a field model is needed to determine $\mathrm{V}\left(\mathrm{x}_{\mathrm{e}}\right)$. The remaining parameters enter equation $(10)$ in the dimensionless ratio $\tau=q E R / k T_{0}$. In Figure 3 we plot $p_{e, R} / p_{\infty} v s . V_{d} / V\left(x_{e}\right)$ for different values of $\tau$. For $\tau$ large, (e.g. $>50$ ), the pressure ratio remains near unity for $\mathrm{V}_{\mathrm{o}} \mathrm{V}<50$; plasma sheet convection is reasonably well represented by the two dimensional treatment even for considerable compression. For $\tau=2$, finite tail effects are important when $V_{0} N>4$; for $\tau=$ 10 , the pressure drops to half that in the infinite tail at $V_{0} / V=$ 15 .

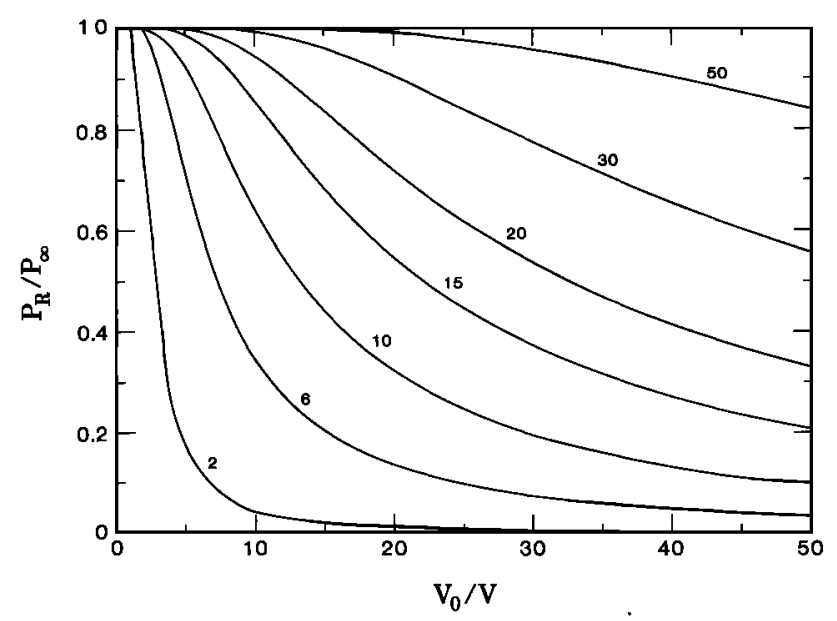

Fig. 3. The ratio $\left(\mathrm{p}_{\mathrm{e}, \mathrm{R}} / \mathrm{p}_{\infty}\right)$ of the plasma pressure along the midnight meridian in a tail of width $2 \mathrm{R}$ to the pressure in a tail of infinite width vs. the ratio ( $\mathrm{Vo} / \mathrm{V})$ of the flux tube volumes at the source location and close to the earth. The curves are labeled by $\mathrm{qER} / \mathrm{kT}_{\mathrm{o}}$, the ratio of the potential drop across half of the tail to the thermal energy per unit charge in the source distribution.

\section{Choice of parameters}

Figure 3 shows that the values of $p_{\infty} / p_{e, R}$ at fixed $V_{\alpha} / V$ are extremely sensitive to the dimensionless parameter $\tau$. What are the relevant values of $\tau$, i.e. of $E, R$, and $k_{0}$ ? For the cross-tail potential, ER, critical to the evaluation of (9) and (10), we select values typical of low levels of $\mathrm{K}_{\mathrm{P}}$ [e.g., Kivelson, 1976; Cowley, 1982], taking it to be of order 15 to $20 \mathrm{kV}$. The corresponding range of $E$ is 0.06 to $0.1 \mathrm{mV} / \mathrm{m}$ for tails of $30-40 R_{E}$ width. E-85 selects the cross tail potential drop as $50 \mathrm{kV}$, which we consider characteristic of moderately active times.

The data on the temperature of the down-tail plasma source are limited. At lunar distances, Rich et al. [1973] find ion temperatures in the range 1 to $5 \mathrm{keV}$ with an average of 2.5 $\mathrm{keV}$. Wolf (personal communication) informs us that E-85 used values from 0.75 to $1.8 \mathrm{keV}$ and $\tau$ values from 14 to 33 . We think that $\tau$ less than 10 is a better choice, consistent with 
the above-mentioned measurements. For a compression factor $\mathrm{V}_{\delta} / \mathrm{V}=30$ and the $\mathrm{E}-85$ values for $\tau$, finite tail corrections give $\mathrm{p} / \mathrm{p}_{\infty} \sim 0.35$. For the same $\mathrm{V}_{\mathrm{o}} / \mathrm{V}$ and $\tau$ of 6 or 2 , the finite tail pressures are much smaller, i.e., $\mathrm{p} / \mathrm{p}_{\infty} \sim 0.1$ or 0.01 .

\section{Comparison with observations}

The variation of pressure with $x_{\mathbf{e}}$ for selected values of $\tau$ is plotted in Figure 2. At large distances, the normalized pressures for different tail widths are indistinguishable. Significant differences appear inside of $25 R_{E}$ where for $\tau \geq 10$, the predicted $\mathrm{p}_{\infty}$ far exceeds $\mathrm{P}_{\mathrm{L}}$ and the negligibly small magnetic curvature forces cannot balance the excess plasma pressure. Thus for large $\tau$, the question framed by EW-80, whether the tail can maintain steady convection, remains unanswered. For realistic low activity values of $\tau(\tau=2.5$ to 3.5) $\mathrm{p}_{\mathrm{e}, \mathrm{R}}$ balances $\mathrm{P}_{\mathrm{L}}$ within the uncertainty ( say, 30\%) in our knowledge of field properties tailward of $20 \mathrm{R}_{\mathrm{E}}$; steady convection should proceed without difficulty. For small $\tau$, the pressure of plasma convected from the distant tail is insufficient to balance magnetic pressure, but the missing pressure can be provided by plasma convected from sources ignored in this treatment. As well, the 2-D tail approximation becomes increasingly inadequate near $10 R_{E}$.

\section{Discussion and Summary}

We have shown that there is little reason to believe that steady convection creates any stress-balance problems in the quiet magnetotail between 30 and $60 R_{\mathrm{E}}$. Magnetic pressure obtained from fits to the lobe magnetic field balances plasma sheet pressure derived from field models using equations (9) and $(10)$ to within the uncertainties of the fits and the models. Inside of $30 \mathrm{R}_{\mathrm{E}}$ we find that the plasma pressure does not become unacceptably large if the finite width of the magnetotail is taken into account and if the critical ratio, $\tau$, of the potential drop across half the tail to the source temperature is selected appropriately for quiet times. Large pressures develop in the near tail if $\tau>10$, as is expected at disturbed times. EW-80 and E-85 selected parameters appropriate to disturbed times ( $\tau=14$ and 33 ) and obtained plasma pressures growing so rapidly near $10 R_{E}$ that they exceeded the magnetic stresses; it seemed unlikely that steady convection could be maintained. We have shown that if $\tau$ is of order 5 or less, the pressure does not exceed the magnetic pressure by more than $30 \%$, which we believe to be within the uncertainty of the field models. We believe that $\tau<5$ is characteristic of relatively quiet times. The possibility remains [Erickson and Wolf, 1980] that for relatively rapid convection or for a very cold plasma source in the distant tail, i.e., for $\tau>10$, unbalanced plasma and magnetic stresses can develop in the near tail and may trigger the onset of substorms.

Acknowledgements. We thank Raymond J. Walker for initiating us into the mysteries of magnetic field models. R. A. Wolf and $J$. Birn provided constructive criticism. This work was supported by the Space Physics program of the National Aeronautics and Space Administration under grant NGL-05007-004 and by the Division of Atmospheric Sciences of the National Science Foundation under grant ATM 86-10858.

UCLA Institute of Geophysics and Planetary Physics Publication Number 3181

\section{References}

Beard, D. B.. The magnetotail magnetic field, J. Geophys. Res., 84, 2118, 1979.
Behannon, K. W., Mapping of the Earth's bow shock and magnetic tail by Explorer 33, J. Geophys. Res., 73, 907, 1968.

Cowley, S. W. H., The causes of convection in the earth's magnetosphere - A review of developments during the IMS, Rev. Geophys. Space Phys., 20, 531, 1982.

Erickson, G. M., On the cause of $x$-line formation in the nearearth plasma sheet: Results of adiabatic convection of plasma sheet plasma, in Magnetic Reconnection, Geophys. Monogr. Series, 30, (E. W. Hones, ed.), pp. 296-302, AGU, Washington, D. C., 1984.

Erickson, G. M., Modeling of plasma-sheet convection: Implications for substorms, $\mathrm{Ph}$. D. dissertation, Rice Univ., Houston, Tex., 1985.

Erickson, G. M., and R. A. Wolf, Is steady state convection possible in the earth's magnetosphere?, Geophys. Res. Lett., 6, 897, 1980.

Hau, L. -N., R. A. Wolf, G. H. Voigt, and C. C. Wu, Steadystate magnetic field configurations for the Earth's magnetotail, J. Geophys. Res., 93, in press, 1988.

Kivelson, M. G., Magnetospheric electric fields and their variation with geomagnetic activity, $\underline{\text { Rev. Geophys. Space }}$ Phys., 14, 189, 1976.

Mihalov, J. D., and C. P. Sonett, The cislunar tail gradient in 1967, J. Geophys. Res., 73, 6837, 1968.

Mihalov, D. S. Colburn, R. G. Currie, and C. P. Sonett, Configuration and reconnection of the geomagnetic tail, $\underline{\mathrm{J}}$. Geophys. Res., 73, 943, 1968.

Olson, W. P., and $\vec{K}$. Pfitzer, A quantitative model of the magnetospheric magnetic field, I. Geophys. Res., 79, 3739, 1974.

Rich, F. J., D. L. Reasoner, and W. J. Burke, Plasma-sheet at lunar distance: characteristics and interaction with the lunar surface, J. Geophys. Res., 78, 8097, 1973.

Schindler, $K$. and $J$. Birn, Self-consistent theory of timedependent convection in the earth's magnetotail, J. Geophys. Res., 87, 2263, 1982.

Spence, H. E., M.G. Kivelson, and R. J. Walker, Static magnetic field models consistent with nearly isotropic plasma pressure, Geophys. Res. Lett., 14, 872, 1987.

Spence, H. E., M.G. Kivelson, R. J. Walker, and D. J. McComas, Magnetotail plasma pressures: Observations, I. Geophys. Res., submitted, 1988a.

Spence, H. E., M. G. Kivelson, and R. J. Walker, Magnetotail plasma pressures: Empirical models and implications for magnetotail stability, J. Geophys. Res., submitted, 1988b.

Tsyganenko, N. A., On the convective mechanism for formation of the plasma sheet in the magnetospheric tail, Planet. Space Sci., 30, 1007, 1982.

Tsyganenko, N. A., Global quantitative models of the geomagnetic field in the cislunar magnetosphere for different disturbance levels, Planet. Space Sci., $\underline{35}, 1347$, 1987.

Tsyganenko, N. A., and A. V. Usmanov, Determination of the magnetospheric current system parameters and development of experimental geomagnetic field models based on data from IMP and HEOS satellites, Planet. Space Sci., 30, 985, 1982.

Voigt, G.-H., A mathematical magnetospheric field model with independent physical parameters, Planet. Space Sci., 29, 1, 1981.

M. G. Kivelson and H. E. Spence, Institute of Geophysics and Planetary Physics, University of California, Ios Angeles, CA 90024-1567. 